ESAIM: PROCEEDINGS, December 2008, Vol. 25, p. 29-43

E. Cancès, S. Faure, B. Graille, Editors

\title{
WELL-POSEDNESS OF AN EPIDEMIOLOGICAL PROBLEM DESCRIBED BY AN EVOLUTION PDE
}

\author{
A. Perasso ${ }^{1,2}$ AND B. Laroche ${ }^{2}$
}

\begin{abstract}
This paper investigates the well-posedness for a non linear transport equation system that models the spread of prion diseases in a managed flock. Existence and uniqueness of solutions are proved with the use of semigroup theory in the case of a Lipschitz perturbation and presence of boundary conditions. Finally, the characteristics of the transport part of the equations allow us to give an implicit expression of the solution.
\end{abstract}

Résumé. Dans ce papier, nous établissons le caractère bien posé d'un problème de transport non linéaire modélisant la propagation d'une maladie à prion dans un troupeau expérimental. Nous prouvons existence et unicité de la solution du problème à l'aide de la théorie des semigroupes, avec présence de conditions de bord et une partie non linéaire localement lipschitzienne. Nous donnons pour conclure une expression implicite de la solution utilisant les caractéristiques des équations de transport.

\section{INTRODUCTION}

The prion pathologies are characterized by a long incubation period, relative to lifespan, during which the disease cannot be detected. At the end of the incubation period, animals develop distinctive clinical signs which are rapidly followed by death. The confounding effects of incubation, natural mortality and the changing force of infection make direct analysis difficult. A mathematical model of the within-population transmission dynamics provides a flexible tool for combining epidemiological and demographic phenomenon.

In this paper, we study the well-posedness of a problem of propagation of a prion disease in a managed population. The model we consider takes into account the population dynamics as well as the spread of the outbreak. Owing to the long incubation period, population demography and management must be included in the model. The mathematical model is therefore formulated in terms of population densities structured according to disease status (Susceptible and Infected), to age and to infection load for infected animals. This leads to a nonlinear integro partial differential dynamical system of transport type.

The first part of the paper is dedicated to the description of the model. In a second part, we establish the well-posedness of the problem. In order to reach that goal we start with the study of the linear part of the problem with the use of semigroup theory. Then we conclude for our initial problem adapting arguments developed in [10].

\footnotetext{
${ }^{1}$ Laboratoire de Mathématiques, Bâtiment 425, Université Paris-Sud XI, F-91405 Orsay Cedex, France.

${ }^{2}$ Laboratoire des signaux et systèmes (L2S) Supélec - 3 rue Joliot-Curie 91192 Gif-sur-Yvette cedex, France.
}

(C) EDP Sciences, SMAI 2008 


\section{Description of the MOdel}

To take into account human management of the population, we assume that age $a$ belongs to a finite interval $[0, A]$, animals reaching age $A$ being systematically culled because they are considered as too old. The population of infected animals is also structured according to the infection load variable $\theta$ that lies in interval $[0,1]$. The densities of Susceptible and Infected are denoted as $S(t, a)$ and $I(t, a, \theta)$, and the number of infected animals at time $t$ is denoted

$$
K(I)(t):=\int_{0}^{A} \int_{0}^{1} I(t, a, \theta) d \theta d a .
$$

The model we use is a simplified version inspired from an epidemic model in [12]. It is a modified version of the classical Kermack-McKendrick $S I$ epidemiological PDE model [6-8] or [9]. The underlying assumptions are homogeneous mixing between all the individuals and a constant probability transmission per contact, $\beta>0$, giving a net rate of infection of $\beta S K(I)$. This is known as the mass action assumption, and comes from a classical microscopic modeling by birth-death processes [5].

Incubation time heterogeneity is modeled through the infection load variable $\theta$. It is assumed that $\theta$ grows exponentially with time with an infection load velocity $c>0$. Infected animals with infection load less than 1 cannot be detected, although they are infectious. An infection load equal to 1 corresponds to the onset of clinical signs and immediate death, either caused by the disease or by culling.

When infection occurs, an animal gets an initial infection load $\left.\theta_{0} \in\right] 0,1[$, so that the incubation time $\tau$ is given by

$$
\tau=-\frac{1}{c} \ln \theta_{0}
$$

Variable incubation time in the infected population is therefore represented by a probability density function (pdf) $\Theta$ of the initial infection load that satisfies

$$
\Theta \in \mathcal{A}(0,1), \quad \Theta(0)=\Theta(1)=0
$$

where $\mathcal{A}(0,1)$ is the set of real-analytic functions on $] 0,1[$ continuous on $[0,1]$. The role of the pdf $\Theta$ is to attribute an initial infection load, and therefore an incubation time, to susceptible animals when they get infected. Such an approach is related to the so-called "size-structured models" encountered in cellular population dynamics (see [1] for a review). An alternative option would be to structure the infected population according to the age of infection like in $[2,3]$. Whatever the parametrization, it leads to a distributed delay structure. Densities $S$ and $I$ satisfy the following transport equations for $(t, a, \theta) \in[0,+\infty[\times[0, A] \times[0,1]$ :

$$
\begin{aligned}
& \frac{\partial S}{\partial t}+\frac{\partial S}{\partial a}=-\mu S-\beta S K(I), \\
& \frac{\partial I}{\partial t}+\frac{\partial I}{\partial a}+\frac{\partial(c \theta I)}{\partial \theta}=-\mu I+\beta \Theta S K(I) .
\end{aligned}
$$

Parameter $\mu>0$ in the model is the basic disease free mortality rate. Infected animals have a strictly positive infected load and we assume in this model that there is no vertical (in utero) transmission. Consequently we associate to the equations (Evol1)-(Evol2) the following boundary conditions, where $t \mapsto n(t)$ represents the birth function :

$$
\left\{\begin{array}{l}
S(t, 0)=n(t), \\
I(t, 0, \theta)=0, \quad I(t, a, 0)=0,
\end{array} \quad \text { for }(t, a, \theta) \in[0,+\infty[\times[0, A] \times[0,1] .\right.
$$

The initial conditions are given by

$$
\left\{\begin{array}{l}
S(0, a)=S_{0}(a), \\
I(0, a, \theta)=I_{0}(a, \theta),
\end{array} \quad \text { for }(a, \theta) \in[0, A] \times[0,1] .\right.
$$


In the following, we will denote $(\mathcal{P})$ the problem

$$
\left\{\begin{array}{c}
(\text { Evol } 1)-(\text { Evol2 }) \\
(B c)-(I c)
\end{array}\right.
$$

\section{WELl-POSEDNESS OF THE PROBLEM}

The Problem $(\mathcal{P})$ is a nonlinear Cauchy problem with a boundary condition, that can be viewed as a perturbation of a linear problem. Consequently, we prove the existence and uniqueness of solutions taking into account a non zero boundary condition. First, we use the ideas of [4] in order to delete the boundary condition and to reduce our problem to a Cauchy problem. Then we adapt the arguments of [10] to prove existence and uniqueness of mild solutions of this Cauchy problem in the positive cone of a Banach space of real valued functions. Finally we deduce the existence and uniqueness of mild solutions for our initial problem $(\mathcal{P})$ and conclude about the well-posedness.

\subsection{Lifting of boundary conditions}

We perform a change of variables in order to transform the boundary condition $(B c)$ into a null condition. For a Banach space $\left(E,\|\cdot\|_{E}\right)$ of real valued functions and any set $F \subset E, F^{+}$shall denote the subset of positive functions of $F$. We also set $X=\mathrm{L}^{2}(0, A) \times \mathrm{L}^{2}((0, A) \times(0,1))$ and we denote $\|\cdot\|_{S}$, and $\|\cdot\|_{I}$, the norm in $\mathrm{L}^{2}(0, A)$, respectively in $\mathrm{L}^{2}((0, A) \times(0,1))$, and $\|\cdot\|_{X}:=\|\cdot\|_{S}+\|\cdot\|_{I}$ the product norm on $X$. We suppose that $\left(S_{0}, I_{0}\right) \in \mathrm{L}^{2}(0, A)^{+} \times \mathrm{L}^{2}((0, A) \times(0,1))^{+}$and $n \in \mathrm{C}_{\mathrm{pw}}\left(\left[0,+\infty[)^{+}\right.\right.$, where $\mathrm{C}_{\mathrm{pw}}([0,+\infty[)$ denotes the set of piecewise continuous functions on $\mathbb{R}^{+}$.

We consider $B:\left[0,+\infty\left[\rightarrow \mathcal{L}\left(\mathrm{C}_{\mathrm{pw}}(0,+\infty), \mathrm{L}^{2}(0, A)\right)\right.\right.$ defined for all $g \in \mathrm{C}_{\mathrm{pw}}(0,+\infty)$ by

$$
(B(t) g)(a):=\left\{\begin{array}{l}
g(0) e^{-\mu t} \quad \text { for } a \in[0, A], a \geq t, \\
g(t-a) e^{-\mu a} \quad \text { for } a \in[0, A], a \leq t,
\end{array}\right.
$$

and the new system

$$
\begin{aligned}
& \frac{\partial \tilde{S}}{\partial t}=-\frac{\partial \tilde{S}}{\partial a}-\mu \tilde{S}-\beta(\tilde{S}+B(t) n) K(\tilde{I}), \\
& \frac{\partial \tilde{I}}{\partial t}=-\frac{\partial \tilde{I}}{\partial a}-\frac{\partial(c \theta \tilde{I})}{\partial \theta}-\mu \tilde{I}+\beta \Theta(\tilde{S}+B(t) n) K(\tilde{I}),
\end{aligned}
$$

with boundary condition

$$
\left\{\begin{array}{l}
\tilde{S}(t, 0)=0, \\
\tilde{I}(t, 0, \theta)=\tilde{I}(t, a, 0)=0,
\end{array} \quad \forall(t, a, \theta) \in[0, T] \times[0, A] \times[0,1],\right.
$$

and initial condition

$$
\left\{\begin{array}{l}
\tilde{S}(0, a)=\tilde{S}_{0}(a)=S_{0}(a)-n(0), \quad \\
\tilde{I}(0, a, \theta)=\tilde{I}_{0}(a, \theta)=I_{0}(a, \theta),
\end{array} \quad \forall(a, \theta) \in[0, A] \times[0,1] .\right.
$$

Remark 2.1. For all $g \in \mathrm{C}_{\mathrm{pw}}(0,+\infty), B(t) g$ satisfies the following transport equation

$$
\frac{\partial B(t) g}{\partial t}+\frac{\partial B(t) g}{\partial a}=-\mu B(t) g
$$


and for $t>0, B(t) g(0)=g(t)$.

Consequently, the transformation $S \mapsto \tilde{S}:=S-B(t) n$ implies that $\tilde{S}$ satisfies equations ( $\tilde{E} v o l 1)-(\tilde{E} v o l 2)$ with conditions $(\tilde{B} c)-(\tilde{I} c)$ iff $S$ satisfies Problem $(\mathcal{P})$.

Let $\Phi_{S}$ and $\Phi_{I}$ be the differential operators defined by

$$
\begin{aligned}
\Phi_{S}: D\left(\Phi_{S}\right) & \rightarrow \mathrm{L}^{2}(0, A), & \Phi_{I}: D\left(\Phi_{I}\right) & \rightarrow \mathrm{L}^{2}((0, A) \times(0,1)), \\
f & \mapsto-f^{\prime}-\mu f, & f & \mapsto-\partial_{a} f-\partial_{\theta} f-(\mu+c) f,
\end{aligned}
$$

where $D\left(\Phi_{S}\right)$ and $D\left(\Phi_{I}\right)$ are the following sets :

$$
\begin{aligned}
& D\left(\Phi_{S}\right):=\left\{f \in \mathrm{C}^{1}[0, A], f(0)=0\right\}, \\
& D\left(\Phi_{I}\right):=\left\{f \in \mathrm{C}^{1}([0, A] \times[0,1]), f(\cdot, 0)=f(0, \cdot)=0\right\} .
\end{aligned}
$$

Setting $\tilde{u}(t):=(\tilde{S}(t, \cdot), \tilde{I}(t, \cdot)), \tilde{u}_{0}:=\left(\tilde{S}_{0}, \tilde{I}_{0}\right), \Phi: D(\Phi) \rightarrow X$ where $D(\Phi):=D\left(\Phi_{S}\right) \times D\left(\Phi_{I}\right) \subset X$ is given by

$$
\Phi:=\left(\begin{array}{cc}
\Phi_{S} & 0 \\
0 & \Phi_{I}
\end{array}\right)
$$

and

$$
\tilde{P}:\left[0,+\infty\left[\times X \rightarrow X, \quad\left(t,\left(\tilde{u}_{S}, \tilde{u}_{I}\right)\right) \mapsto\left(\begin{array}{c}
\tilde{P}_{S} \\
\tilde{P}_{I}
\end{array}\right):=\left(\begin{array}{c}
-\beta\left(\tilde{u}_{S}+B(t) n\right) K\left(\tilde{u}_{I}\right) \\
\beta \Theta\left(\tilde{u}_{S}+B(t) n\right) K\left(\tilde{u}_{I}\right)
\end{array}\right),\right.\right.
$$

well-posedness of equations ( $\tilde{E} v o l 1)-(\tilde{E} v o l 2)$ with initial condition $(\tilde{I} c)$ is equivalent to well-posedness of the following problem

$$
(\tilde{\mathcal{P}}) \quad\left\{\begin{array}{c}
\frac{d}{d t} \tilde{u}(t)=\Phi \tilde{u}(t)+\tilde{P}(t, \tilde{u}(t)) \\
\tilde{u}(0)=\tilde{u}_{0} .
\end{array}\right.
$$

\subsection{The linear problem}

This section is devoted to the definition of the semigroups generated by the differential operator $\Phi$ for the linear problem.

Proposition 2.2. $\Phi_{S}$ and $\Phi_{I}$ are infinitesimal generators of two strongly continuous positive semigroups of bounded linear operators, $T_{S}:\left[0,+\infty\left[\rightarrow \mathcal{L}\left(\mathrm{L}^{2}(0, A)\right)\right.\right.$ and $T_{I}:\left[0,+\infty\left[\rightarrow \mathcal{L}\left(\mathrm{L}^{2}((0, A) \times(0,1))\right)\right.\right.$, defined by

$$
\left(T_{S}(t) f\right)(a):=\left\{\begin{array}{cc}
f(a-t) e^{-\mu t} & \text { for } a \geq t, \\
0 & \text { for } a \leq t,
\end{array} \quad \text { for all } f \in \mathrm{L}^{2}(0, A),\right.
$$

and

$$
\left(T_{I}(t) f\right)(a, \theta):=\left\{\begin{array}{cl}
f\left(a-t, \theta e^{-c t}\right) e^{-(\mu+c) t} & \text { for } a \geq t, \\
0 & \text { for } a \leq t,
\end{array} \quad \text { for all } f \in \mathrm{L}^{2}((0, A) \times(0,1)) .\right.
$$

We have moreover the following estimation :

$$
\sup \left(\left\|T_{S}(t)\right\|,\left\|T_{I}(t)\right\|\right) \leq e^{-\mu t} \quad \forall t \geq 0 .
$$

Proof. We can easily check that $\left(T_{S}(t)\right)_{t \geq 0}$ and $\left(T_{I}(t)\right)_{t \geq 0}$ are semigroups of bounded strongly continuous operators.

In order to prove that $\Phi_{I}$, respectively $\Phi_{S}$, is the infinitesimal generator of $T_{I}$, respectively $T_{S}$, we prove that

$$
\lim _{t \rightarrow 0}\left\|\frac{T_{I}(t) f-f}{t}-\Phi_{I} f\right\|_{I}=0 .
$$


For $f \in D\left(\Phi_{I}\right)$ and for $t>0$,

$$
\begin{array}{r}
\left\|\frac{T_{I}(t) f-f}{t}-\Phi_{I} f\right\|_{I}^{2} \leq(A-t) \sup _{(a, \theta) \in[t, A] \times[0,1]}\left|\frac{\left(T_{I}(t) f\right)(a, \theta)-f(a, \theta)}{t}-\Phi_{I} f(a, \theta)\right|^{2} \\
+\int_{0}^{t} \int_{0}^{1}\left|\frac{f(a, \theta)}{t}-\Phi_{I} f(a, \theta)\right|^{2} d \theta d a .
\end{array}
$$

The last term in the right hand side of (6) can be bounded as follows :

$$
\begin{array}{r}
\int_{0}^{t} \int_{0}^{1}\left|\frac{f(a, \theta)}{t}-\Phi_{I} f(a, \theta)\right|^{2} d \theta d a \leq 2 \int_{0}^{t} \int_{0}^{1}\left|\frac{f(a, \theta)}{a}\right|^{2} d \theta d a \\
+2 t \sup _{(a, \theta) \in[0, A] \times[0,1]}\left|\Phi_{I} f(a, \theta)\right|^{2}
\end{array}
$$

Since $f \in D\left(\Phi_{I}\right)$, using the Lebesgue dominated convergence theorem in (7) we have

$$
\lim _{t \rightarrow 0} \int_{0}^{t} \int_{0}^{1}\left|\frac{f(a, \theta)}{t}-\Phi_{I} f(a, \theta)\right|^{2} d \theta d a=0 .
$$

Let $f \in D\left(\Phi_{I}\right)$ and the function $G$ defined on the subset $\mathcal{E}_{I}:=\{(t, a, \theta) \in[0,+\infty[\times[0, A] \times[0,1], a \geq t\}$ by :

$$
G(t, a, \theta):=\left\{\begin{array}{cc}
\frac{f\left(a-t, \theta e^{-c t}\right) e^{-(\mu+c) t}-f(a, \theta)}{t} & t \neq 0 \\
\Phi_{I} f(a, \theta) & t=0 .
\end{array}\right.
$$

We can see in Proposition 3.1 of appendix, that $G$ is continuous on $\mathcal{E}_{I}$ and then is uniformly continuous. Consequently,

$$
\lim _{t \rightarrow 0} \sup _{(a, \theta) \in[t, A] \times[0,1]}\left|\frac{\left(T_{I}(t) f\right)(a, \theta)-f(a, \theta)}{t}-\Phi_{I} f(a, \theta)\right|=0,
$$

and we conclude using the limits in (8) and (9) reported in (6). Similar considerations prove that $\Phi_{S}$ is the infinitesimal generator of $T_{S}$.

Estimation (5) as well as the positivity of $T_{S}$ and $T_{I}$ are easily derived from their expressions.

We now define the concept of mild solution, and give the two main results of the paper related to well-posedness.

Definition 2.3. A mild solution of Problem $(\tilde{\mathcal{P}})$ is a function $\tilde{u} \in C([0,+\infty[, X)$ that satisfies

$$
\tilde{u}(t)=T(t) \tilde{u}_{0}+\int_{0}^{t} T(t-s) \tilde{P}(s, \tilde{u}(s)) d s \quad \forall t \in[0, T] .
$$

The following result holds,

Theorem 2.4. Suppose that $S_{0} \in \mathrm{L}^{2}(0, A)^{+}, I_{0} \in \mathrm{L}^{2}((0, A) \times(0,1))^{+}$and $n \in \mathrm{C}_{\mathrm{pw}}(0,+\infty)^{+}$. Then the problem $(\tilde{\mathcal{P}})$ has a unique mild solution $\tilde{u}:=(\tilde{S}, \tilde{I})$ such that $\tilde{I}(t) \geq 0$ and $\tilde{S}(t)+B(t) n \geq 0$ for all $t \geq 0$.

Consequently,

Corollary 2.5. If $n \in \mathrm{C}_{\mathrm{pw}}(0,+\infty)^{+}$, then for all $u_{0}:=\left(S_{0}, I_{0}\right) \in \mathrm{L}^{2}(0, A)^{+} \times \mathrm{L}^{2}((0, A) \times(0,1))^{+}$the Problem $(\mathcal{P})$ has a unique mild solution $u:=(S, I) \in \mathrm{C}([0,+\infty[, X)$ in the following sense :

$$
S(t, a)=\left\{\begin{array}{l}
\left(T_{S}(t) S_{0}\right)(a)+\int_{0}^{t}\left(T_{S}(t-s) P_{S}(u(s))\right)(a) d s \quad \text { for } a \geq t \\
(B(t) n)(a)+\int_{0}^{t}\left(T_{S}(t-s) P_{S}(u(s))\right)(a) d s \quad \text { for } a \leq t
\end{array}\right.
$$


and

$$
I(t, a, \theta)=\left(T_{I}(t) I_{0}\right)(a, \theta)+\int_{0}^{t}\left(T_{I}(t-s) P_{I}(u(s))\right)(a, \theta) d s,
$$

where $S(t)$ and $I(t)$ are positive functions for all $t \in[0,+\infty[$.

Moreover, for all $T>0$ and all $u_{0}, v_{0} \in \mathrm{L}^{2}(0, A)^{+} \times \mathrm{L}^{2}((0, A) \times(0,1))^{+}$, there exists $K>0$ such that the associated mild solutions $u, v$ satisfy

$$
\|u(t)-v(t)\|_{X} \leq K\left\|u_{0}-v_{0}\right\|_{X} \quad \forall t \in[0, T] .
$$

The next section is devoted to the proof of these main results. In order to prove them, we start with the study of the non-linear semi-group.

\subsection{The nonlinear problem}

A consequence of Proposition 2.2 is that for all $\tilde{u}_{0} \in D\left(\Phi_{S}\right) \times D\left(\Phi_{I}\right)$, the linear problem associated to $(\tilde{\mathcal{P}})$ has a unique classical solution given by $\tilde{u}(t)=T(t) \tilde{u}_{0}$ for $t \geq 0$. For $\tilde{u}_{0} \in X$, we now look for a mild solution of $(\tilde{\mathcal{P}})$ as the sum of the solution of the linear problem and a term accounting for the perturbation $\tilde{P}$. To this end, we need a local control of the perturbation $\tilde{P}$. This is done in the following proposition.

Proposition 2.6. The perturbation $\tilde{P}$ is a locally Lipschitz function in $u=\left(u_{S}, u_{I}\right)$, uniformly in $t$ on segments of $\mathbb{R}^{+}:$for all $r>0$ and all $T>0$ there exists $m(r, T)>0$ such that

$$
\left\|\tilde{P}\left(t, u_{1}\right)-\tilde{P}\left(t, u_{2}\right)\right\|_{X} \leq m(r, T)\left\|u_{1}-u_{2}\right\|_{X} \quad \forall u_{1}, u_{2} \in B_{X}(0, r), \forall t \in[0, T] .
$$

Moreover, $r \mapsto m(r, T)$ is a growing continuous function.

Proof. Let $T, r>0$ and $u_{1}:=\left(u_{S_{1}}, u_{I_{1}}\right), u_{2}:=\left(u_{S_{2}}, u_{I_{2}}\right)$ such that $\left(u_{1}, u_{2}\right) \in\left(B_{X}(0, r)\right)^{2}$. Then for all $t \in[0, T]$ we have

$$
\begin{aligned}
\left\|\tilde{P}\left(t, u_{1}\right)-\tilde{P}\left(t, u_{2}\right)\right\|_{X} & =\left\|\beta\left(u_{S_{2}}+B(t)(n)\right) K\left(u_{I_{2}}\right)-\beta\left(u_{S_{1}}+B(t)(n)\right) K\left(u_{I_{1}}\right)\right\|_{S} \\
& +\left\|\beta \Theta\left(u_{S_{1}}+B(t) n\right) K\left(u_{I_{1}}\right)-\beta \Theta\left(u_{S_{2}}+B(t) n\right) K\left(u_{I_{2}}\right)\right\|_{I} .
\end{aligned}
$$

Using expression of $K(I)$ and Cauchy-Schwarz inequality we have

$$
\begin{gathered}
\left\|\beta\left(u_{S_{2}}+B(t) n\right) K\left(u_{I_{2}}\right)-\beta\left(u_{S_{1}}+B(t) n\right) K\left(u_{I_{1}}\right)\right\|_{S}^{2} \leq 2 \beta^{2}\left\|\left(u_{S_{2}}+B(t) n\right) K\left(u_{I_{1}}-u_{I_{2}}\right)\right\|_{S}^{2} \\
+2 \beta^{2}\left\|\left(u_{S_{1}}-u_{S_{2}}\right) K\left(u_{I_{1}}\right)\right\|_{S}^{2} \\
\leq 2 \beta^{2} A\left(\left\|u_{S_{2}}+B(t) n\right\|_{S}^{2}\left\|u_{I_{1}}-u_{I_{2}}\right\|_{I}^{2}\right. \\
\left.+\left\|u_{I_{1}}\right\|_{I}^{2}\left\|u_{S_{1}}-u_{S_{2}}\right\|_{S}^{2}\right) .
\end{gathered}
$$

Since for all $t \in[0, T]$

$$
\|B(t) n\|_{S}^{2}=\int_{0}^{\min (t, A)}|n(t-a)|^{2} e^{-2 \mu a} d a+\int_{\min (t, A)}^{A}|n(0)|^{2} e^{-2 \mu t} d a \leq\|n\|_{\mathrm{L}^{2}(0, T)}^{2}+A|n(0)|^{2},
$$

we obtain for all $t \in[0, T]$ the following upper bounds

$$
\begin{aligned}
\| \beta\left(u_{S_{2}}+B(t) n\right) K\left(u_{I_{2}}\right)- & \beta\left(u_{S_{1}}+B(t) n\right) K\left(u_{I_{1}}\right) \|_{S}^{2} \leq \\
& 2 \beta^{2} A\left(\left(r+\sup _{t \in[0, T]}\|B(t) n\|_{S}\right)^{2}\left\|u_{I_{1}}-u_{I_{2}}\right\|_{I}^{2}+r^{2}\left\|\left(S_{1}-S_{2}\right)\right\|_{S}^{2}\right),
\end{aligned}
$$


and setting $c(r, T):=2 \beta^{2} A\left(r+\sup _{t \in[0, T]}\|B(t) n\|_{S}^{2}\right)$, we deduce

$$
\left\|\beta\left(u_{S_{2}}+B(t) n\right) K\left(u_{I_{2}}\right)-\beta\left(u_{S_{1}}+B(t) n\right) K\left(u_{I_{1}}\right)\right\|_{S}^{2} \leq c(r, T)\left\|u_{1}-u_{2}\right\|_{X}^{2} .
$$

We also have for all $t \in[0, T]$

$$
\left\|\beta \Theta\left(u_{S_{1}}+B(t) n\right) K\left(u_{I_{1}}\right)-\beta \Theta\left(u_{S_{2}}+B(t) n\right) K\left(u_{I_{2}}\right)\right\|_{I}^{2} \leq c(r, T)\|\Theta\|_{\infty}^{2}\left\|u_{1}-u_{2}\right\|_{X}^{2} .
$$

Using (14) we can conclude, setting $m(r, T):=\sqrt{c(r, T)\left(1+\|\Theta\|_{\infty}^{2}\right)}$, that

$$
\left\|\tilde{P}\left(t, u_{1}\right)-\tilde{P}\left(t, u_{2}\right)\right\|_{X} \leq m(r, T)\left\|u_{1}-u_{2}\right\|_{X} .
$$

We can easily check that $r \mapsto m(r, T)$ is a growing function using definitions of $c(r, T)$ and $m(r, T)$.

We now prove the Theorem $2.4 \mathrm{using}$ a fixed point method adapting the proof of [11]. Consider $T>0$, $r:=2\left(\left\|u_{0}\right\|_{X}+\|n\|_{L^{2}(0, T)}+\sup _{t \in[0, T]}\|B(t) n\|_{S}\right)$ and $\delta>0$ such that

$$
\delta<\min \left\{T, \frac{1}{2 m(r, T)}, \frac{1}{\beta \sqrt{A}\left(\|n\|_{\mathrm{L}^{2}(0, T)}^{2}+\left\|S_{0}\right\|_{S}^{2}\right)^{\frac{1}{2}}+m(r, T)}\right\} .
$$

We define the mapping $F: C([0, \delta], X) \rightarrow C([0, \delta], X)$ by

$$
F \tilde{u}(t):=\left(\begin{array}{c}
F_{S} \tilde{u}(t) \\
F_{I} \tilde{u}(t)
\end{array}\right):=\left(\begin{array}{c}
g(t)-B(t) n \\
T_{I}(t) \tilde{I}_{0}+\int_{0}^{t} T_{I}(t-s) \tilde{P}_{I}(s, \tilde{u}(s)) d s
\end{array}\right)
$$

where

$$
g(t): a \mapsto \begin{cases}S_{0}(a-t) e^{-\left(\mu t+\beta \int_{0}^{t} K(\tilde{I})(s) d s\right)} & \text { if } a \geq t, \\ n(t-a) e^{-\left(\mu a+\beta \int_{t-a}^{t} K(\tilde{I})(s) d s\right)} & \text { if } a \leq t .\end{cases}
$$

Lemma 2.7. The mapping $F$ preserves the closed subset $\mathcal{F}$ defined by

$$
\mathcal{F}:=\left\{\tilde{u}:=(\tilde{S}, \tilde{I}) \in C([0, \delta], X), \sup _{t \in[0, \delta]}\|\tilde{u}(t)\|_{X} \leq r, \tilde{S}(t)+B(t) n \geq 0 \text { and } \tilde{I}(t) \geq 0\right\}
$$

Moreover $F$ is a contraction mapping on $\mathcal{F}$.

Proof. Let $\tilde{u} \in \mathcal{F}$. Since $\left\|T_{I}(t)\right\| \leq 1$,

$$
\begin{aligned}
\|F \tilde{u}(t)\|_{X} & \leq\left\|S_{0}\right\|_{S}+\|n\|_{\mathrm{L}^{2}(0, T)}+\sup _{t \in[0, T]}\|B(t) n\|_{S}+\left\|\tilde{I}_{0}\right\|_{I}+ \\
& \quad \int_{0}^{t}\left\|T_{I}(t-s)\right\|\left(\left\|\tilde{P}_{I}(s, \tilde{u}(s))-\tilde{P}_{I}(s, 0)\right\|_{I}+\left\|\tilde{P}_{I}(s, 0)\right\|_{I}\right) d s \\
& \leq\left\|u_{0}\right\|_{X}+\|n\|_{\mathrm{L}^{2}(0, T)}+\sup _{t \in[0, T]}\|B(t) n\|_{S}+\int_{0}^{t}(r m(r, T)+0) d s \\
& \leq\left\|u_{0}\right\|_{X}+\|n\|_{\mathrm{L}^{2}(0, T)}+\sup _{t \in[0, T]}\|B(t) n\|_{S}+\operatorname{tr} m(r, T),
\end{aligned}
$$

and using definition of $\delta$ and $r$

$$
\|F \tilde{u}(t)\|_{X} \leq r
$$


Moreover, we can easily check that $F_{S} \tilde{u}(t)+B(t) n \geq 0$, and then $\tilde{P}_{I}(s, \tilde{u}(s)) \geq 0$. Since $\tilde{I}_{0} \geq 0$ and $T_{I}$ is a positive semigroup, we can conclude $F_{I} \tilde{u}(t) \geq 0$ and $F$ preserves $\mathcal{F}$. Now, let us prove that $F$ is a contraction mapping. We can see in Proposition 3.2 in appendix that for $\tilde{u}_{1}, \tilde{u}_{2} \in \mathcal{F}$,

$$
\left\|F_{S} \tilde{u}_{1}(t)-F_{S} \tilde{u}_{2}(t)\right\|_{S} \leq \beta \sqrt{A} t\left(\left\|S_{0}\right\|_{S}^{2}+\|n\|_{\mathrm{L}^{2}(0, T)}^{2}\right)^{\frac{1}{2}} \sup _{t \in[0, \delta]}\left\|\tilde{u}_{1}(t)-\tilde{u}_{2}(t)\right\|_{X} \quad \forall t \in[0, \delta] .
$$

Furthermore,

$$
\begin{aligned}
\left\|F_{I} \tilde{u}_{1}(t)-F_{I} \tilde{u}_{2}(t)\right\|_{I} & \leq \int_{0}^{t}\left\|\tilde{P}_{I}\left(s, \tilde{u}_{1}(s)\right)-\tilde{P}_{I}\left(s, \tilde{u}_{2}(s)\right)\right\|_{I} d s \\
& \leq m(r, T) t \sup _{t \in[0, \delta]}\left\|\tilde{u}_{1}(t)-\tilde{u}_{2}(t)\right\|_{X} \quad \forall t \in[0, \delta] .
\end{aligned}
$$

With (16) and (17) we have for all $t \in[0, \delta]$

$$
\sup _{t \in[0, \delta]}\left\|F \tilde{u}_{1}(t)-F \tilde{u}_{2}(t)\right\|_{X} \leq\left(m(r, T)+\beta \sqrt{A}\left(\left\|S_{0}\right\|_{S}^{2}+\|n\|_{\mathrm{L}^{2}(0, T)}^{2}\right)^{\frac{1}{2}}\right) \delta \sup _{t \in[0, \delta]}\left\|\tilde{u}_{1}(t)-\tilde{u}_{2}(t)\right\|_{X},
$$

which guarantees that $F$ is a contraction mapping on $\mathcal{F}$.

Consequently to lemma $2.7, F$ has a unique fixed point $\tilde{u}=(\tilde{S}, \tilde{I})$ in $\mathcal{F}$. We now prove that this solution can be extended to $[0, T]$.

Let us suppose that $\tilde{u}$ is defined on some interval $[0, \tau], 0<\tau<T$. As in Proposition 3.3 of appendix we consider $\tilde{u}_{\tau}(t)=\left(\tilde{S}_{\tau}(t), \tilde{I}_{\tau}(t)\right)$ the unique solution on $\left[\tau, \tau+\delta^{\tau}\right]$ of

$$
\tilde{u}_{\tau}(t)=\left(\begin{array}{c}
g_{\tau}(t)-B(t) n \\
T_{I}(t-\tau) \tilde{I}(\tau)+\int_{\tau}^{t} T_{I}(t-s) \tilde{P}_{I}\left(s, \tilde{u}_{\tau}(s)\right) d s
\end{array}\right)
$$

with

and

$$
\begin{gathered}
g_{\tau}(t): a \mapsto\left\{\begin{array}{l}
g\left(\tau, a-(t-\tau) e^{-\left(\mu(t-\tau)+\beta \int_{\tau}^{t} K\left(\tilde{I}_{\tau}\right)(s) d s\right)} \quad \text { if } a \geq t-\tau,\right. \\
n(t-a) e^{-\left(\mu a+\beta \int_{t-a}^{t} K\left(\tilde{I}_{\tau}\right)(s) d s\right)} \quad \text { if } a \leq t-\tau,
\end{array}\right. \\
\delta^{\tau}<\min \left\{T-\tau, \frac{1}{2 m\left(r^{\tau}, T\right)}, \frac{1}{\beta \sqrt{A}\left(\|n\|_{\mathrm{L}^{2}(0, T)}^{2}+\|\tilde{S}(\tau)+B(\tau) n\|_{S}^{2}\right)^{\frac{1}{2}}+m\left(r^{\tau}, T\right)}\right\},
\end{gathered}
$$

$$
r^{\tau}:=2\left(\left\|S_{0}\right\|_{S}+\|n\|_{\mathrm{L}^{2}(0, T)}+\|\tilde{I}(\tau)\|_{I}+\sup _{t \in[0, T]}\|B(t) n\|_{S}\right) .
$$

We also prove in Proposition 3.3 of appendix that the function $\tilde{u}$ can be extended to $\left[0, \tau+\delta^{\tau}\right]$ defining $\tilde{u}:=\tilde{u}_{\tau}$ on $\left[\tau, \tau+\delta_{\tau}\right]$. Clearly $\tilde{u}$ is in $\mathrm{C}\left(\left[0, \tau+\delta^{\tau}\right], X\right)$.

Let $J \subset[0, T]$ be the maximal interval of existence of the solution $\tilde{u}$, and let us denote $t_{\max }:=\sup J$. We can check that there exists a constant $C_{T}>0$ independent of $t_{\max }$ such that for all $t \in J$

$$
\|\tilde{u}(t)\|_{X} \leq C_{T} .
$$

Indeed, using expression of $g_{\tau}$ and (18), we can easily check that

$$
\tilde{S}(t, a)=g(t, a)-(B(t) n)(a) \quad \forall(t, a) \in J \times[0, A],
$$

and then for all $t \in J$

$$
\|\tilde{S}(t)\|_{S} \leq\left\|S_{0}\right\|_{S}+\|n\|_{\mathrm{L}^{2}(0, T)}+\sup _{t \in[0, T]}\|B(t) n\|_{S}
$$


For $\tilde{I}$ we have

Using the following estimations

$$
\|\tilde{I}(t)\|_{I} \leq\left\|\tilde{I}_{0}\right\|_{I}+\int_{0}^{t}\left\|T_{I}(t-s)\right\|\|\tilde{P}(s, \tilde{u}(s))\|_{I} d s .
$$

$$
\begin{aligned}
\left\|\tilde{P}_{I}(t, \tilde{u}(t))\right\|_{I} & \leq \beta\|\Theta\|_{\mathrm{L}^{2}(0,1)}\|g(t)\|_{S} \sqrt{A}\|\tilde{I}(t)\|_{I} \\
& \leq \beta \sqrt{A}\|\Theta\|_{\mathrm{L}^{2}(0,1)}\left(\left\|S_{0}\right\|_{S}+\|n\|_{\mathrm{L}^{2}(0, T)}\right)\|\tilde{I}(t)\|_{I},
\end{aligned}
$$

we can deduce, denoting $c:=\beta \sqrt{A}\|\Theta\|_{\mathrm{L}^{2}(0,1)}\left(\left\|S_{0}\right\|_{S}+\|n\|_{\mathrm{L}^{2}(0, T)}\right)$, that for all $t \in J$

$$
\|\tilde{I}(t)\|_{I} \leq\left\|\tilde{I}_{0}\right\|_{I}+c \int_{0}^{t}\|\tilde{I}\|_{I} d s
$$

By Gronwall's inequality we finally obtain

$$
\|\tilde{I}(t)\|_{I} \leq\left\|\tilde{I}_{0}\right\|_{I} e^{c t} \quad \forall t \in J
$$

Consequently to (22) and (23), we deduce (20) with $C_{T}=\left\|\tilde{I}_{0}\right\|_{I} e^{c T}+\left\|S_{0}\right\|_{S}+\|n\|_{\mathrm{L}^{2}(0, T)}+\sup _{t \in[0, T]}\|B(t) n\|_{S}$. This uniform bound on the solutions guarantees the existence of a global-in-time solution on $[0, T]$, as stated in [10] or [11]. Moreover, clearly $\tilde{u} \in \mathrm{C}([0, T], X)$ and satisfies $\tilde{I}(t) \geq 0$ and $\tilde{S}(t)+B(t) n \geq 0$ for all $t \in[0, T]$.

We now finish the proof of Theorem 2.4 with the two following lemmas.

Lemma 2.8. $\tilde{u}$ is a mild solution of Problem $(\tilde{\mathcal{P}})$.

Proof. Equation (10) is satisfied for $\tilde{I}$ by definition. For $a \geq t$ we have

$$
\begin{aligned}
T_{S}(t) \tilde{S}_{0}(a) & +\int_{0}^{t}\left(T_{S}(t-s) \tilde{P}_{S}(s, \tilde{u}(s))\right)(a) d s \\
& =\tilde{S}_{0}(a-t) e^{-\mu t}-\int_{0}^{t} \beta g(s, a+s-t) e^{-\mu(t-s)} K(\tilde{I})(s) d s \\
& =\tilde{S}_{0}(a-t) e^{-\mu t}-S_{0}(a-t) e^{-\mu t} \int_{0}^{t} \beta K(\tilde{I})(s) e^{-\beta \int_{0}^{s} K(\tilde{I})(u) d u} d s \\
& =S_{0}(a-t) e^{-\left(\mu t+\beta \int_{0}^{t} K(\tilde{I})(s) d s\right)}-S_{0}(a-t) e^{-\mu t}+\tilde{S}_{0}(a-t) e^{-\mu t} \\
& =g(t, a)-(B(t) n)(a),
\end{aligned}
$$

and finally for $a \geq t$

$$
\left(T_{S}(t) \tilde{S}_{0}\right)(a)+\int_{0}^{t}\left(T_{S}(t-s) \tilde{P}_{S}(s, \tilde{u}(s))\right)(a) d s=\tilde{S}(t, a)
$$

For $a \leq t$, we can check that $\left(T_{S}(t) \tilde{S}_{0}\right)(a)=0$ and

$$
\int_{0}^{t}\left(T_{S}(t-s) \tilde{P}(s, \tilde{u}(s))\right)(a) d s=-\int_{t-a}^{t} \beta g(s, a+s-t) e^{-\mu(t-s)} K(I)(s) d s .
$$

Using the definition of $g$ given in (15) we obtain

$$
\begin{aligned}
\int_{0}^{t}\left(T_{S}(t-s) \tilde{P}(s, \tilde{u}(s))\right)(a) d s & =-n(t-a) e^{-\mu a} \int_{t-a}^{t} \beta K(I)(s) e^{-\beta \int_{t-a}^{s} K(I)(u) d u} d s \\
& =n(t-a) e^{-\mu a}\left(e^{-\beta \int_{t-a}^{t} K(I)(u) d u}-1\right) .
\end{aligned}
$$


Consequently, for $a \leq t$ we have

$$
\int_{0}^{t}\left(T_{S}(t-s) \tilde{P}(s, \tilde{u}(s))(a) d s=g(t, a)-(B(t) n)(a),\right.
$$

and we obtain (24) for $a \leq t$. Finally, $\tilde{u}(t)$ clearly satisfies initial condition $(\tilde{I} c)$, then is a mild solution of $(\tilde{\mathcal{P}})$ on $[0, T]$.

Lemma 2.9. For any initial value $\tilde{u}_{0} \in X$, there is a unique mild solution $\tilde{u}$ defined on $[0, T]$ that satisfies $\tilde{u}(0)=\tilde{u}_{0}$. Moreover, for all $\tilde{u}_{0}, \tilde{v}_{0} \in X$, the associated solutions $\tilde{u}, \tilde{v}$ satisfy

$$
\|\tilde{u}(t)-\tilde{v}(t)\|_{X} \leq e^{m(r, T) T}\left\|\tilde{u}_{0}-\tilde{v}_{0}\right\|_{X}
$$

where $r:=\max _{t \in[0, T]}\left(\|\tilde{u}(t)\|_{X},\|\tilde{v}(t)\|_{X}\right)$.

The proof of Lemma 2.9 and the uniqueness of the solution on $[0,+\infty]$ is a classical result developped in [10].

We now conclude for existence and uniqueness of the solution of the initial problem $(\mathcal{P})$ stated in Corollary 2.5. We denote $P$ the perturbation in (Evol1)-(Evol2) given by

$$
P: X \rightarrow X, \quad u=\left(u_{S}, u_{I}\right) \mapsto\left(\begin{array}{c}
P_{S}(u) \\
P_{I}(u)
\end{array}\right):=\left(\begin{array}{c}
-\beta u_{S} K\left(u_{I}\right) \\
\beta \Theta u_{S} K\left(u_{I}\right)
\end{array}\right) .
$$

Let $\tilde{u}$ be the unique mild solution that satisfies the Problem $(\tilde{\mathcal{P}})$, and let us denote for all $t \in[0,+\infty[$

$$
u(t)=(S(t), I(t)):=\tilde{u}(t)+(B(t) n, 0) \in \mathrm{L}^{2}(0, A) \times \mathrm{L}^{2}((0, A) \times(0,1)) .
$$

Clearly $u(0)=u_{0}$ and $S(t)$ and $I(t)$ are positive functions for all $t \in[0,+\infty[$. We can easily see in the proof of Theorem 2.4 that $I$ satisfies (12). We can also check that $S(t, a)=g(t, a)$, and equalities (24) and (25) in proof of Lemma 2.8 imply that (11) is satisfied.

Let us prove the continuity of $t \mapsto u(t)$. Equality $I=\tilde{I}$ implies that $I$ is in $\mathrm{C}\left(\left[0,+\infty\left[, \mathrm{L}^{2}((0, A) \times(0,1))\right)\right.\right.$. Since $S(t)=\tilde{S}(t)+B(t) n$ and $\tilde{S}$ is in $\mathrm{C}\left(\left[0,+\infty\left[, \mathrm{L}^{2}(0, A)\right)\right.\right.$, we just have to prove that $t \mapsto B(t) n$ is a function of $\mathrm{C}\left(\left[0,+\infty\left[, \mathrm{L}^{2}(0, A)\right)\right)\right.$ to conclude for the continuity of $t \mapsto S(t)$.

Let $t_{0} \in\left[0,+\infty\left[\right.\right.$. Then for any $t>t_{0}$ we have

$$
\begin{aligned}
& \int_{0}^{A}\left|(B(t) n)(a)-\left(B\left(t_{0}\right) n\right)(a)\right|^{2} d a=\int_{0}^{\min \left(t_{0}, A\right)}\left|n(t-a) e^{-\mu a}-n\left(t_{0}-a\right) e^{-\mu a}\right|^{2} d a \\
& +\int_{\min \left(t_{0}, A\right)}^{\min (t, A)}\left|n(t-a) e^{-\mu a}-n(0) e^{-\mu t_{0}}\right|^{2} d a+\int_{\min (t, A)}^{A}\left|n(0) e^{-\mu t}-n(0) e^{-\mu t_{0}}\right|^{2} d a .
\end{aligned}
$$

Let us study the behavior of the three terms of the second member for $t$ close to $t_{0}$. Consequently to the Lebesgue dominated convergence theorem it is clear that

$$
\lim _{t \rightarrow t_{0}} \int_{0}^{\min \left(t_{0}, A\right)}\left|n(t-a) e^{-\mu a}-n\left(t_{0}-a\right) e^{-\mu a}\right|^{2} d a=0 .
$$

We next have

$$
\int_{\min (t, A)}^{A}\left|n(0) e^{-\mu t}-n(0) e^{-\mu t_{0}}\right|^{2} d a \leq A|n(0)|^{2}\left|e^{-\mu t}-e^{-\mu t_{0}}\right|^{2}
$$


and consequently with a change of variables

$$
\begin{gathered}
\int_{\min \left(t_{0}, A\right)}^{\min (t, A)}\left|n(t-a) e^{-\mu a}-n(0) e^{-\mu t_{0}}\right|^{2} d a=\int_{t-\min (t, A)}^{t-\min \left(t_{0}, A\right)}\left|n(a) e^{-\mu(t-a)}-n(0) e^{-\mu t_{0}}\right|^{2} d a \\
\leq 2 \int_{t-\min (t, A)}^{t-\min \left(t_{0}, A\right)}|n(a)-n(0)|^{2} d a+8|n(0)|^{2}\left(\min (t, A)-\min \left(t_{0}, A\right)\right) .
\end{gathered}
$$

With (29) and the upper bounds (30) and (31) we conclude that

$$
\lim _{\substack{t \rightarrow t_{0} \\ t>t_{0}}} \int_{0}^{A}\left|(B(t) n)(a)-\left(B\left(t_{0}\right) n\right)(a)\right|^{2} d a=0 .
$$

We can easily obtain the same limit for $t<t_{0}$, and then $t \mapsto B(t) n$ is in $\mathrm{C}\left(\left[0,+\infty\left[, \mathrm{L}^{2}(0, A)\right)\right.\right.$. Also holds for $S$, and $u=(S, I) \in \mathrm{C}([0,+\infty[, X)$.

Finally, using initial and boundary conditions $(\tilde{I} c)-(\tilde{B} c)$ and definition $(28)$ of $(S, I)$, we conclude that for $\left(S_{0}, I_{0}\right) \in D(\Phi), S$ and $I$ satisfy the conditions $(I c)-(B c)$, and then the problem $(\mathcal{P})$.

With Lemma 2.9 and definition of $u$ we conclude that $(13)$ is satisfied with $K=e^{m(r, T) T}$. Consequently the mild solution, given an initial condition, is unique.

Corollary 2.10. For all $t \in\left[0,+\infty\left[, S\right.\right.$ and I satisfy in $\mathrm{L}^{2}(0, A)$ and in $\mathrm{L}^{2}((0, A) \times(0,1))$ respectively:

$$
\begin{aligned}
& S(t, a)=\left\{\begin{array}{l}
S_{0}(a-t) e^{-\left(\mu t+\beta \int_{0}^{t} K(I)(s) d s\right)} \quad \text { for } a \geq t, \\
n(t-a) e^{-\left(\mu a+\beta \int_{t-a}^{t} K(I)(s) d s\right)} \quad \text { for } a \leq t,
\end{array}\right. \\
& I(t, a, \theta)=\left\{\begin{array}{c}
I_{0}\left(a-t, \theta e^{-c t}\right) e^{-(\mu+c) t} \\
+S_{0}(a-t) e^{-\mu t} \int_{0}^{t} e^{c(s-t)} \Theta\left(\theta e^{c(s-t)}\right) \beta K(I)(s) e^{-\beta \int_{0}^{s} K(I)(u) d u} d s \\
\text { for } a \geq t, \\
n(t-a) e^{-\mu a} \int_{t-a}^{t} e^{c(s-t)} \Theta\left(\theta e^{c(s-t)}\right) \beta K(I)(s) e^{-\beta \int_{t-a}^{s} K(I)(u) d u} d s \\
\text { for } a \leq t .
\end{array}\right.
\end{aligned}
$$

Proof. Since $S(t, a)=g(t, a)$ then $S$ satisfies (32). Moreover, we just have to use expression of $T_{I}, P_{I}$ and substitute $S$ by its expression (32) in the equation (12) to conclude that $I$ also satisfies (33).

Remark 2.11. The expressions of $S$ and $I$ in Corollary 2.10 can be viewed as a consequence of a change of variables using the characteristics of the transport part of the PDEs. These characteristics are given by

$$
\left\{\begin{array}{l}
\dot{a}=1, \\
\dot{\theta}=c \theta,
\end{array}\right.
$$

which suggests the change of variables $(t, a, \theta) \mapsto(t, b, d)$ where

$$
\begin{aligned}
& a=t-b, \\
& \theta=e^{c(t-d)} .
\end{aligned}
$$

This change of variable has the following biological meaning : the variable $b$ denotes the birth date and the variable $d$ denotes the death date.

Formulating the problem in the base $(t, b, d)$, the PDEs (Evol1)-(Evol2) become differential equations with differentiation w.r.t $t$, that can be integrated in equations (32)-(33). 

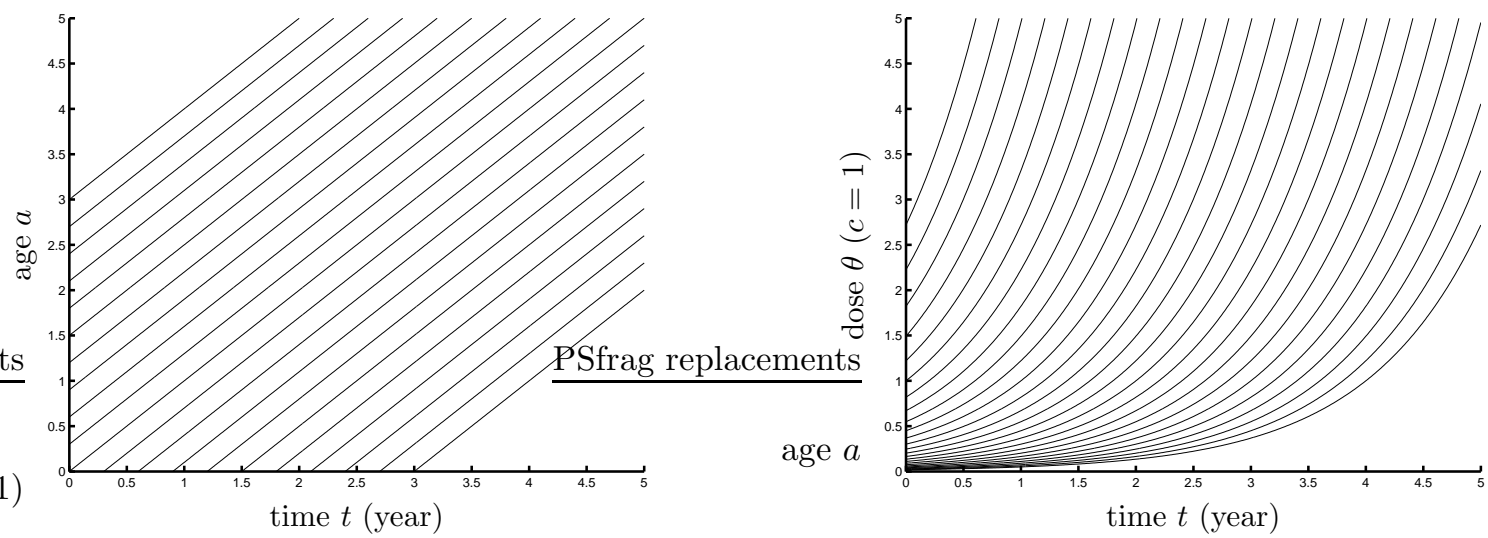

FiguRE 1. Characteristic curves

\section{Conclusion}

The mathematical analysis performed in this paper is a necessary step before tackling parameter estimation on real life data. This type of structured population model is indeed representative of biological phenomenon since it is a simplified version of a multi-genotype model of scrapie transmission that has already been used in [13]. The results presented in this paper are currently beeing extended to the multi-genotype case for further parameter estimation.

\section{APPENDIX}

Proposition 3.1. Let $f \in D\left(\Phi_{I}\right)$. The function $G$ defined by

$$
G(t, a, \theta):=\left\{\begin{array}{cc}
\frac{f\left(a-t, \theta e^{-c t}\right) e^{-(\mu+c) t}-f(a, \theta)}{t} & t \neq 0, \\
\Phi_{I} f(a, \theta) & t=0,
\end{array}\right.
$$

is continuous on $\mathcal{E}_{I}=\{(t, a, \theta) \in[0,+\infty[\times[0, A] \times[0,1], a \geq t\}$.

Proof. Clearly $\Phi_{I}\left(D\left(\Phi_{I}\right)\right) \subset \mathrm{C}((0, A] \times[0,1])$, then $G$ is continuous outside the line $t=0$ and on the line $t=0$. Let us prove that $G$ is continuous at the junction.

We consider a sequence $\left\{\left(t_{n}, a_{n}, \theta_{n}\right)\right\}_{n \geq 0}$ of $\mathcal{E}_{I}$ such that

$$
\lim _{n \rightarrow+\infty}\left(t_{n}, a_{n}, \theta_{n}\right)=(0, a, \theta) \in \mathcal{E}_{I},
$$

and we denote by $\varphi$ the smooth map $\varphi:(t, a, \theta) \mapsto\left(a-t, \theta e^{-c t}\right)$. The function $f \circ \varphi$ is $\mathrm{C}^{1}$, and Taylor approximation gives

$$
(f \circ \varphi)\left(t_{n}, a_{n}, \theta_{n}\right)=(f \circ \varphi)\left(0, a_{n}, \theta_{n}\right)+D_{\left(0, a_{n}, \theta_{n}\right)}(f \circ \varphi)\left(t_{n}, 0,0\right)+o\left(\left|t_{n}\right|\right),
$$

that we can write, using expressions of $D_{\varphi\left(0, a_{n}, \theta_{n}\right)} f$ and $D_{\left(0, a_{n}, \theta_{n}\right)} \varphi$,

$$
f\left(a_{n}-t_{n}, \theta_{n} e^{-c t_{n}}\right)-f\left(a_{n}, \theta_{n}\right)=-\partial_{a} f\left(a_{n}, \theta_{n}\right) t_{n}-c \theta_{n} e^{-c t_{n}} \partial_{\theta} f\left(a_{n}, \theta_{n}\right) t_{n}+o\left(\left|t_{n}\right|\right) .
$$


Since $f \in D\left(\Phi_{I}\right)$, we have

$$
\lim _{n \rightarrow+\infty} \frac{f\left(a_{n}-t_{n}, \theta_{n} e^{-c t_{n}}\right)-f\left(a_{n}, \theta_{n}\right)}{t_{n}}=-\partial_{a} f(a, \theta)-c \theta \partial_{\theta} f(a, \theta)
$$

and we can conclude for the continuity by noticing that

$$
G\left(t_{n}, a_{n}, \theta_{n}\right)=e^{-(\mu+c) t_{n}} \frac{f\left(a_{n}-t_{n}, \theta_{n} e^{-c t_{n}}\right)-f\left(a_{n}, \theta_{n}\right)}{t_{n}}+f\left(a_{n}, \theta_{n}\right) \frac{e^{-(\mu+c) t_{n}}-1}{t_{n}}
$$

which converges on

$$
-\partial_{a} f(a, \theta)-c \theta \partial_{\theta} f(a, \theta)-(\mu+c) f(a, \theta)=G(0, a, \theta) .
$$

Proposition 3.2. For all $\tilde{u}_{1}, \tilde{u}_{2} \in \mathcal{F}$, we have

$$
\left\|F_{S} \tilde{u}_{1}(t)-F_{S} \tilde{u}_{2}(t)\right\|_{S} \leq \sqrt{A} \beta t\left(\left\|S_{0}\right\|_{S}^{2}+\|n\|_{\mathrm{L}^{2}(0, T)}^{2}\right)^{\frac{1}{2}} \sup _{t \in[0, \delta]}\left\|\tilde{u}_{1}(t)-\tilde{u}_{2}(t)\right\|_{X} \quad \forall t \in[0, \delta] .
$$

Proof. Using the inequality $\left|e^{-x}-e^{-y}\right| \leq|x-y|$ for $x, y \geq 0$ and expression of $K(I)$ we obtain

$$
\begin{aligned}
\left\|F_{S} \tilde{u}_{1}(t)-F_{S} \tilde{u}_{2}(t)\right\|_{S}^{2} & \leq \int_{0}^{\min (t, A)}\left|\beta n(t-a) \int_{t-a}^{t} K\left(\tilde{I}_{1}-\tilde{I}_{2}\right)(u) d u\right|^{2} d a \\
& +\int_{\min (t, A)}^{A}\left|\beta S_{0}(a-t) \int_{0}^{t} K\left(\tilde{I}_{1}-\tilde{I}_{2}\right)(u) d u\right|^{2} d a .
\end{aligned}
$$

Using Cauchy-Schwarz we have $K\left(\tilde{I}_{1}-\tilde{I}_{2}\right)(u) \leq \sqrt{A}\left\|\tilde{I}_{1}-\tilde{I}_{2}\right\|_{I}$, and for $a \leq t$

$$
\left|\int_{t-a}^{t} K\left(\tilde{I}_{1}-\tilde{I}_{2}\right)(u) d u\right| \leq t \sqrt{A} \sup _{t \in[0, \delta]}\left\|\tilde{I}_{1}-\tilde{I}_{2}\right\|_{I}
$$

and for $t \geq a$

$$
\left|\int_{0}^{t} K\left(\tilde{I}_{1}-\tilde{I}_{2}\right)(u) d u\right| \leq t \sqrt{A} \sup _{t \in[0, \delta]}\left\|\tilde{I}_{1}-\tilde{I}_{2}\right\|_{I}
$$

Then

$\| F_{S}\left(\tilde{u}_{1}(t)-F_{S} \tilde{u}_{2}(t) \|_{S}^{2} \leq t^{2} \beta^{2} A\left(\int_{0}^{\min (t, A)}|n(t-a)|^{2} d a+\int_{\min (t, A)}^{A}\left|S_{0}(a-t)\right|^{2} d a\right)\left(\sup _{t \in[0, \delta]}\left\|\tilde{I}_{1}-\tilde{I}_{2}\right\|_{I}\right)^{2}\right.$

and (16) consequently.

Proposition 3.3. Let $\tilde{u} \in \mathrm{C}([0, \tau], X)$ satisfying $\tilde{u}(t)=F \tilde{u}(t)$ on $[0, \tau]$. Then $\tilde{u}$ can be extended on $\left[0, \tau+\delta^{\tau}\right]$ where

$$
\delta^{\tau}<\min \left\{T-\tau, \frac{1}{2 m\left(r^{\tau}, T\right)}, \frac{1}{\beta \sqrt{A}\left(\|n\|_{L^{2}(0, T)}^{2}+\|\tilde{S}(\tau)+B(\tau) n\|_{S}^{2}\right)^{\frac{1}{2}}+m\left(r^{\tau}, T\right)}\right\}
$$

with $r^{\tau}:=2\left(\left\|S_{0}\right\|_{S}+\|n\|_{\mathrm{L}^{2}(0, T)}+\|\tilde{I}(\tau)\|_{I}+\sup _{t \in[0, T]}\|B(t) n\|_{S}\right)$. 
Proof. We denote $\tilde{u}=(\tilde{S}, \tilde{I})$ the solution on $[0, \tau]$. We define the mapping

$$
F^{\tau}: \mathrm{C}\left(\left[\tau, \tau+\delta^{\tau}\right], X\right) \rightarrow \mathrm{C}\left(\left[\tau, \tau+\delta^{\tau}\right], X\right),
$$

by

where

$$
F^{\tau} \tilde{u}^{\tau}(t):=\left(\begin{array}{c}
F_{S}^{\tau} \tilde{u}^{\tau}(t) \\
F_{I}^{\tau} \tilde{u}^{\tau}(t)
\end{array}\right):=\left(\begin{array}{c}
g_{\tau}(t)-B(t) n \\
T_{I}(t-\tau) \tilde{I}(\tau)+\int_{\tau}^{t} T_{I}(t-s) \tilde{P}_{I}\left(s, \tilde{u}^{\tau}(s)\right) d s
\end{array}\right)
$$

$$
g_{\tau}(t): a \mapsto\left\{\begin{array}{cl}
(\tilde{S}(\tau, a-(t-\tau))+(B(\tau) n)(a- & (t-\tau))) \\
\times e^{-\left(\mu(t-\tau)+\beta \int_{\tau}^{t} K\left(\tilde{I}_{\tau}\right)(s) d s\right)} & \text { if } a \geq t-\tau, \\
n(t-a) e^{-\left(\mu a+\beta \int_{t-a}^{t} K\left(\tilde{I}_{\tau}\right)(s) d s\right)} & \text { if } a \leq t-\tau .
\end{array}\right.
$$

Let us prove that $F^{\tau}$ preserves the closed subset $\mathcal{F}^{\tau}$ defined by

$$
\mathcal{F}^{\tau}:=\left\{\tilde{u}^{\tau}=\left(\tilde{S}^{\tau}, \tilde{I}^{\tau}\right) \in \mathrm{C}\left(\left[\tau, \tau+\delta^{\tau}\right], X\right),\left\|\tilde{u}^{\tau}\right\|_{X} \leq r^{\tau}, \tilde{S}^{\tau}(t)+B(t) n \geq 0 \text { and } \tilde{I}^{\tau}(t) \geq 0\right\},
$$

and $F^{\tau}$ is a contraction mapping on $\mathcal{F}^{\tau}$.

For $\tilde{u}^{\tau} \in \mathcal{F}^{\tau}$ we have, since $\left\|T_{I}(t)\right\| \leq 1$,

$$
\begin{aligned}
\left\|F^{\tau} \tilde{u}^{\tau}(t)\right\|_{X} & \leq\|g(\tau, a-(t-\tau))\|_{S}+\sup _{t \in[0, T]}\|B(t) n\|_{S}+\|\tilde{I}(\tau)\|_{I}+\int_{\tau}^{t} r^{\tau} m\left(r^{\tau}, T\right) d s \\
& \leq\left\|S_{0}\right\|_{S}+\|n\|_{\mathrm{L}^{2}(0, T)}+\sup _{t \in[0, T]}\|B(t) n\|_{S}+\|\tilde{I}(\tau)\|_{I}+(t-\tau) r^{\tau} m\left(r^{\tau}, T\right),
\end{aligned}
$$

and using the definition of $\delta^{\tau}$,

$$
\|F \tilde{u}(t)\|_{X} \leq r^{\tau}
$$

Moreover, we can check easily $F_{S}^{\tau} \tilde{u}^{\tau}(t)+B(t) n \geq 0$ and then $\tilde{P}_{I}\left(s, \tilde{u}^{\tau}(s)\right) \geq 0$. Since $\tilde{I}_{0} \geq 0$ and $T_{I}$ is a positive semigroup, we can conclude $F_{I}^{\tau} \tilde{u}^{\tau}(t) \geq 0$ and $F^{\tau}$ preserves $\mathcal{F}^{\tau}$. Now, let us prove that $F^{\tau}$ is a contraction mapping. Doing the same than in the proof of Proposition 3.2 in appendix, we can see that for $\tilde{u}_{1}, \tilde{u}_{2} \in \mathcal{F}^{\tau}$ and for all $t \in\left[\tau, \tau+\delta^{\tau}\right]$,

$$
\begin{aligned}
\| F_{S}^{\tau} \tilde{u}_{1}(t) & -F_{S}^{\tau} \tilde{u}_{2}(t) \|_{S} \\
& \leq \sqrt{A} \beta(t-\tau)\left(\|\tilde{S}(\tau)+B(\tau) n\|_{S}^{2}+\|n\|_{\mathrm{L}^{2}(0, T)}^{2}\right)^{\frac{1}{2}} \sup _{t \in\left[\tau, \tau+\delta^{\tau}\right]}\left\|\tilde{u}_{1}(t)-\tilde{u}_{2}(t)\right\|_{X} .
\end{aligned}
$$

Furthermore for all $t \in\left[\tau, \tau+\delta^{\tau}\right]$

$$
\begin{aligned}
\left\|F_{I} \tilde{u}_{1}(t)-F_{I} \tilde{u}_{2}(t)\right\|_{I} & \leq \int_{\tau}^{t}\left\|\tilde{P}_{I}\left(s, \tilde{u}_{1}(s)\right)-\tilde{P}_{I}\left(s, \tilde{u}_{2}(s)\right)\right\|_{I} d s \\
& \leq m\left(r^{\tau}, T\right)(t-\tau) \sup _{t \in\left[\tau, \tau+\delta^{\tau}\right]}\left\|\tilde{u}_{1}(t)-\tilde{u}_{2}(t)\right\|_{X} .
\end{aligned}
$$

With (34) and (35) we have for all $t \in\left[\tau, \tau+\delta^{\tau}\right]$

$$
\begin{array}{r}
\sup _{t \in\left[\tau, \tau+\delta^{\tau}\right]}\left\|F \tilde{u}_{1}(t)-F \tilde{u}_{2}(t)\right\|_{X} \leq\left(m\left(r^{\tau}, T\right)+\sqrt{A} \beta\left(\left\|S_{0}\right\|_{S}^{2}+\|n\|_{L^{2}(0, T)}^{2}\right)^{\frac{1}{2}}\right) \delta \\
\times \sup _{t \in\left[\tau, \tau+\delta^{\tau}\right]}\left\|\tilde{u}_{1}(t)-\tilde{u}_{2}(t)\right\|_{X},
\end{array}
$$

which guarantees $F^{\tau}$ is a contraction mapping on $\mathcal{F}^{\tau}$. Consequently, $F^{\tau}$ has a unique fixed point in $\mathcal{F}^{\tau}$. 


\section{REFERENCES}

[1] O. Arino. A survey of structured cell population dynamics. Acta Biotheoretica, 43:3-25, 1998.

[2] O. Arino, A. Bertuzzi, A. Gandolfi, E. Sánchez, and C. Sinisgalli. A model with 'growth retardation' for the kinetic heterogeneity of tumour cell populations. Math. Biosciences, 206:185-199, 2007.

[3] J. Dyson, R. Villella-Bressa, and G.F. Webb. Asynchronous exponential growth in an age structured population of proliferating and quiescent cells. Math. Biosciences, 177-178:73-83, 2002.

[4] H. O. Fattorini. Boundary control systems. SIAM J. Control \& Opt., 6(3):349-385, 1968

[5] D. Givon, R. Kupferman, and A. Stuart. Extracting macroscopic dynamics : model problems and algorithms. Institute of Physics Publishing, Nonlinearity, 17:R55-R127, 2004.

[6] W. O. Kermack and A. G. McKendrick. A contribution to the mathematical theory of epidemics. Proc. Roy. Soc., A, 115:700$721,1927$.

[7] W. O. Kermack and A. G. McKendrick. Contributions to the mathematical theory of epidemics. II.- The problem of endemicity. Proc. Roy. Soc., A, 138:55-83, 1932.

[8] W. O. Kermack and A. G. McKendrick. Contributions to the mathematical theory of epidemics. III.-Further studies of the problem of endemicity. Proc. Roy. Soc., A, 141:94-122, 1933.

[9] J. D. Murray. Mathematical Biology. Springer, 2002.

[10] A. Pazy. Semigroups of Linear Operators and Applications to Partial Differential Equations. Applied Mathematical Sciences 44. Springer, 1983.

[11] I. Segal. Non-linear semi-groups. Annals of Mathematics, 78:339-364, 1963.

[12] S. M. Stringer, N. Hunter, and M. E. J. Woolhouse. A mathematical model of the dynamics of scrapie in a sheep flock. Math. Biosciences, 153(1-2):79-98, 1998.

[13] S. Touzeau, M. E. Chase-Topping, L. Matthews, D. Lajous, F. Eychenne, N. Hunter, J.D. Foster, G. Simm, J.-M. Elsen, and M. E. J. Woolhouse. Modelling the spread of scrapie in a sheep flock : evidence for increased transmission during lambing seasons. Arch. Vir., 151:735-751, April 2006. 